\title{
Improving quality of science through better animal welfare: the NC3Rs strategy
}

\author{
Mark J Prescott \& Katie Lidster
}

Good animal welfare is linked to the quality of research data derived from laboratory animals, their validity as models of human disease, the number of animals required to reach statistical significance and the reproducibility of in vivo studies. Identifying new ways of understanding and improving animal welfare, and promoting these in the scientific community, is therefore a key part of the work of the National Centre for the Replacement, Refinement and Reduction of Animals in Research (NC3Rs). Our strategy for animal welfare includes funding research to generate an evidence base to support refinements, office-led data sharing to challenge existing practices, events and networks to raise awareness of the evidence base, and the creation of online and other resources to support practical implementation of refinement opportunities.

The use of animals in research is a topic many people feel strongly about. Opinion polls in the UK show that, although the majority of the public can accept animal experimentation (71\%), this acceptance is on the condition that there is no alternative and no unnecessary suffering for the animals ${ }^{1}$. The public also place a high priority on animal welfare: $35 \%$ think that animals should not be used in scientific research on welfare grounds and 54\% want to know more about work to improve research animal welfare. Legislation on the protection of animals used for scientific purposes reflects these societal views and requires that both animal use and suffering be minimized ${ }^{2}$. Research funders too have high expectations in terms of the use and care of animals in funded research, which is reflected in their policies and the terms and conditions of grant awards $s^{3,4}$. There is then wide acceptance of the principle that the welfare of research animals should be optimized, that adverse effects such as pain, fear and distress should be avoided or minimized, and that the animals should be maintained under conditions that promote their health and well-being-this is the refinement principle of the $3 \mathrm{Rs}^{5,6}$.

So far so good, but how well are we, the scientific community, doing at minimizing harm to research animals? Although most institutions that are breeding, housing and using animals in research strive to do their best, there are substantial challenges to achieving widespread implementation of refinement approaches ${ }^{7}$ (Table 1). In many areas, there is a lack of understanding of the effect of scientific procedures on animals and of the evidence base to support refinement and genuine improvements in welfare. Awareness of the existing opportunities for refinement can be variable, and in some cases there is a reluctance to question and challenge established practices and cultures. Staff on the ground may not have the practical tools, resources and support needed to put the current knowledge base into practice. The consequences of failing to address challenges such as these, and to fully implement refinement, are serious, however. They concern not just ethics, the law, and the reputation of and public confidence in animal research, but also the quality, validity and reproducibility of the science derived from the animal subjects.

\section{The importance of good welfare for good quality science}

"Good welfare equals good science" has become the mantra ever since Trevor Poole first highlighted the relationship between research animal welfare and the quality of science ${ }^{8}$, and, in the intervening decades, the evidence base for this relationship has been steadily growing. Animal welfare means how an animal is coping with the conditions in which it lives. An animal is in a good state of welfare if, as indicated by scientific evidence, it is healthy, comfortable, well-nourished, safe, able to express innate behavior, and if it is not suffering from unpleasant states such as pain, fear and distress. Obviously, there are cases in research in which a high degree of suffering may need to occur to achieve the scientific objectives (which would be assessed case-by-case before authorization is granted), but in all cases every effort should be made to minimize unnecessary harm because animals with compromised welfare have disturbed behavior, physiology and immunology. This can lead to unreliable conclusions and/or unwanted variation in scientific output, affecting both the reliability and repeatability

National Centre for the Replacement, Refinement and Reduction of Animals in Research (NC3Rs), London, UK. Correspondence should be addressed to M.J.P. (mark.prescott@nc3rs.org.uk). 


\begin{tabular}{|c|c|}
\hline What we need to understand & What we need to do \\
\hline $\begin{array}{l}\text { The effects of research procedures } \\
\text { on animal welfare }\end{array}$ & $\begin{array}{l}\text { Be motivated to optimize the welfare } \\
\text { of the animals in our care }\end{array}$ \\
\hline $\begin{array}{l}\text { How negative welfare states can } \\
\text { be avoided or alleviated }\end{array}$ & $\begin{array}{l}\text { Put the evidence base into practice, } \\
\text { routinely and uniformly }\end{array}$ \\
\hline $\begin{array}{l}\text { How positive welfare states can } \\
\text { be encouraged }\end{array}$ & $\begin{array}{l}\text { Possess the necessary information, } \\
\text { knowledge and skills }\end{array}$ \\
\hline $\begin{array}{l}\text { How welfare states, and hence } \\
\text { the impact of refinements, can be } \\
\text { reliably measured }\end{array}$ & $\begin{array}{l}\text { Develop and share new ways of } \\
\text { measuring and improving welfare }\end{array}$ \\
\hline $\begin{array}{l}\text { How animal welfare impacts on } \\
\text { quality of science }\end{array}$ & $\begin{array}{l}\text { Demonstrate publicly that welfare } \\
\text { improvements are being made }\end{array}$ \\
\hline
\end{tabular}

of experiments. Throughout this article, we give examples of this link between animal welfare and scientific outcomes as we describe the efforts of the NC3Rs to refine animal research.

\section{The NC3Rs strategy for refinement}

The NC3Rs is an independent scientific organization established by the UK government to discover, develop and promote new ways of replacing, reducing and refining the use of animals in science. Our vision in terms of refinement and animal welfare is to support an effective pipeline of animal welfare research being put into practice to benefit the lifetime experience of research animals and to improve the quality of science. Our strategy to deliver this vision consists of four pillars, detailed below, which balance scientific discovery with implementation (Fig. 1). The approach spans the breadth of the NC3Rs remit, across both industry and academic use of animals, and the range of laboratory animal species and procedures used in the biosciences.

\section{Research funding}

To date, over $£ 8.5$ million of our $£ 50$ million research investment has been for projects aimed primarily at refinement (some awards address more than one ' $R$ '). The robust peer review procedures for our funding schemes (www.nc3rs.org.uk/funding) ensure that the funded research is internationally competitive and focused on priority areas, such as fields in which large numbers of animals are used, that involve procedures causing severe suffering or that use sensitive species. Our awards fall into one of three broad categories (Fig. 2), with the greatest investment being in research to refine the use of rodent models of disease and non-human primate models of human cognition and perception. Examples of recent publications from our refinement research portfolio are given below.

\section{Research to develop and validate new ways of measuring and assessing the welfare of animals and to understand the effect of research procedures on welfare}

How an animal feels is a key component of its welfare. Although behavioral and physiological responses, such as cortisol/ corticosterone levels or heart rate, are good measures of emotional arousal and are widely used as welfare indicators, they can lack

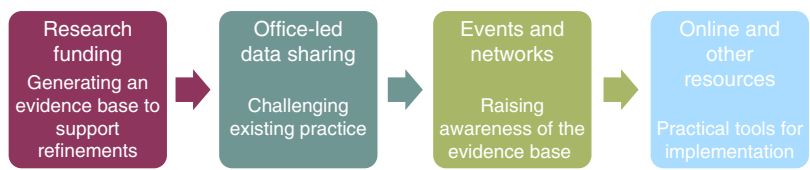

FIGURE 1 | NC3Rs strategy for refinement, balancing scientific discovery with implementation.

valence as both negative states, such as fear, and positive states, such as pleasure, may result in the same responses regardless of the animal's underlying emotion. The cognitive bias approach pioneered by Mike Mendl and Liz Paul at the University of Bristol avoids this problem ${ }^{9,10}$. The basic premise is that animals in a more negative emotional state are more likely to be 'pessimistic' when presented with an ambiguous stimulus (midway between two stimuli they have been trained to recognize as rewarding and non-rewarding) than animals in a more positive state, which display more opportunistic judgments. With NC3Rs funding, Mendl and Paul are developing an automated test of affective state in mice and rats, and other grant holders have successfully applied the cognitive bias approach to assess the emotional state of dogs, rhesus macaques and common marmosets ${ }^{1-13}$.

NC3Rs-funded PhD student Helen Gray and colleagues at Newcastle University recently reported the effects on physiology, behavior and task performance of different fluid restriction protocols in rhesus macaques, which are commonly used to motivate monkeys to perform behavioral and cognitive tasks in neuroscience studies ${ }^{14}$. The research suggests that fluid restriction $(14-26 \mathrm{ml} / \mathrm{kg} / \mathrm{d})$ has less of an effect on animal welfare than previously proposed (although the sample size was small, only four adult males). The macaques were able to concentrate their urine to retain fluid and keep blood values in normal ranges. Bodyweight loss during the working week was regained at the weekend once free access to water was returned. However, some of the observed behavioral changes may be indicative of reduced welfare during fluid control, and so further research is needed on the behavioral and psychological effect of these protocols.

\section{Research to generate an evidence base for improvements in husbandry, such as housing and handling}

The importance of good housing and husbandry is recognized in legislation and guidelines on the use and care of animals in science, but empirical evidence is lacking in some areas. Laura Scullion Hall and colleagues at the University of Stirling and AstraZeneca have recently shown that beagles housed in a purpose-built facility with modern home pen design and exposed to regular staff contact and training demonstrate more signs of positive welfare (for example, resting) and fewer negative signs (for example, vigilance and stereotypies) than do beagles housed in traditional housing ${ }^{15}$. Lottie Hosie, at the University of Chester, found that Xenopus laevis housed in tanks with dark (ecologically relevant) backgrounds showed fewer signs of stress (lower corticosterone release rates in females, a small proportion of atypical locomotor behaviors and snout abrasions in both sexes, and a smaller drop in body mass) than those kept in tanks with white backgrounds ${ }^{16}$. Often, this is the kind of information required by the animal 


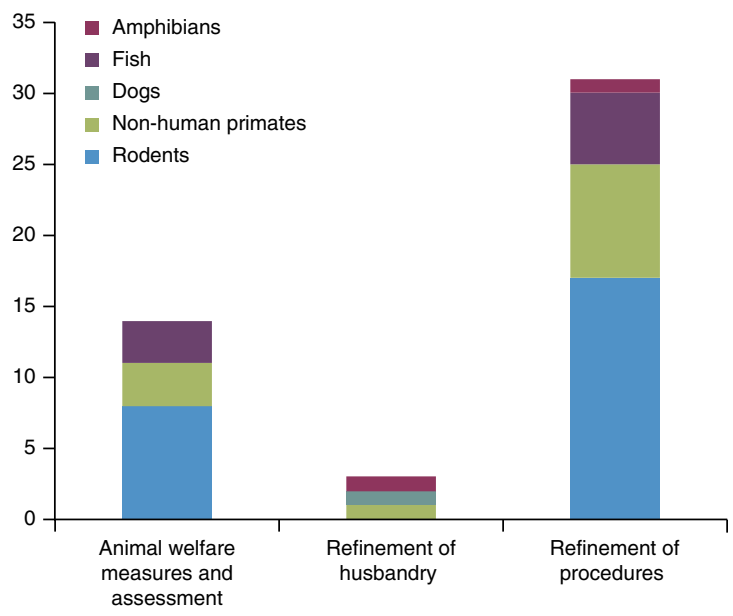

FIGURE 2 | NC3Rs awards primarily aimed at refinement (48 awards), by broad category and species group.

facility senior management team to justify committing funds to improve housing standards.

The quality of our interactions with laboratory animals is also a key determinant of their welfare. Kelly Gouveia and her supervisor Jane Hurst at the University of Liverpool have extended 3Rs prize-winning research on non-aversive handling methods for laboratory mice ${ }^{17,18}$. Traditional capture by the base of the tail leads to high anxiety in mice, as assessed by a range of behavioral measures, whereas the alternatives of using a tunnel or cupped hands leads to voluntary approach, low anxiety and acceptance of physical restraint. These responses generalize across different strains and sexes of mice, and across handlers with differing levels of experience. These now-validated refined handling techniques are available to the scientific community to improve the welfare of the millions of mice used worldwide (for practical guidance on their use, see the video tutorial on the NC3Rs website, http://www. nc3rs.org.uk/mouse-handling-tutorial). However, what is likely to be a driver for researchers to make greater use of the refined techniques is the more recent finding of Gouveia and Hurst on the effect of handling method on test responses. Tail-handled mice showed impaired performance on behavioral phenotyping assays, being much more inactive and reluctant to exploring test arenas or novel objects. Missed observations on account of this tail-handling anxiety could lead to false negatives.

Biotechnology and Biological Sciences Research Council (BBSRC) funded $\mathrm{PhD}$ research, examined by NC3Rs staff, further illustrates the importance for science of refining animal handling and restraint. During her PhD at the University of Stirling, Louisa Tasker worked with cynomolgus macaques that were used in regulatory toxicology studies, where the effects of drugs on cardiovascular and other parameters are measured ${ }^{19}$. Monkeys that had experienced improved restraint techniques and positive socialization with humans showed reduced fear responses to care staff during handling and cardiovascular data collection. Notably, such animals also showed lower baseline heart rate and blood pressure values, and reduced between-animal variation, which meant that drug-induced changes could be identified with greater accuracy.
There were also fewer artifacts in ECG traces from these animals as a result of reduced movement, vocalization and tension, making it easier to determine arrhythmias.

\section{Research to develop and validate new models, procedures or techniques that avoid or reduce pain, suffering, distress or lasting harm}

Challenging the status quo by developing more humane alternatives to long-established research models and practices that carry a high welfare and ethical burden is crucial if we are to make substantial progress in refinement. NC3Rs researchers are at the forefront of this effort. For instance, Michael Emerson and colleagues at Imperial College London have developed a new approach for studying pulmonary embolism in mice ${ }^{20,21}$. Conventional modeling relies on injection of thrombogenic substances in conscious animals that often results in paralysis and death. In contrast, Emerson's refined model is performed under general anesthesia using radiolabeled platelets and imaging to measure platelet function in real time during non-fatal thromboembolism. Not only is the new in vivo model a significant refinement (from 'severe' to 'unclassified' severity) and reduction (using 90\% fewer mice), it also better mimics the physiology and biochemistry of the condition in man and models a broader spectrum beyond the extreme, fatal stage.

Chris Petkov and his Newcastle University colleagues have used techniques from the treatment of cancer patients to develop a new non-invasive means of head immobilization for macaques used in neurobiology studies in which brain activity is monitored ${ }^{22}$. The new approach, consisting of a custom-fitted face mask and helmet, provides an alternative in some studies to surgically implanted head restraint devices, which commonly become infected and can fail over time, leading to animals being removed from the study and data collection being stopped.

In addition to our response-mode funding schemes, we operate a challenge-led innovation scheme called CRACK IT Challenges (http://www.crackit.org.uk). CRACK IT funds the development and commercialization of technologies that benefit the 3Rs and address specific business and scientific challenges identified by industry and academic 'sponsors'. One such challenge, 'Rodent Little Brother', sponsored by MRC Harwell, was to develop a system for automated recording of mouse behavior in the home cage. The solution (ActualHCA) from Actual Analytics is built around a standard individually ventilated cage and comprises a radiofrequency reader baseplate as well as an infrared camera and computer. It enables continuous recording of the location, activity and behavior of individual, micro-chipped mice in established social groups over prolonged periods of time.

The potential of this system for improving phenotyping of genetically altered mice, monitoring of disease progression, implementation of humane endpoints and investigation of social behaviors is tremendous. Currently, most phenotyping studies involve assessment on a battery of tests, conducted over short periods in a novel environment during the inactive period of the mice (our daytime), following their removal from the home cage by human handlers and separation from group mates. All of these factors can cause anxiety or stress, leading to changes in activity 
and behavior that may mask subtle, but important, phenotypes. ActualHCA avoids these problems and provides an incredibly rich data set and massive statistical power. It has already revealed unique insights into the effects of genetic background on individual and group behavior not previously recognized ${ }^{23}$.

\section{Office-led data sharing}

The NC3Rs runs office-led data sharing programs to challenge existing practice and identify new opportunities for refinement. Our expert working groups are led by our team of post-doctoral scientists and comprise relevant international experts with experience in the specific areas of interest. The outputs are published in the peerreviewed literature and are promoted at major scientific conferences and in research organizations. Working groups have focused in areas in which refinement can have a substantial effect, including rodent models of epilepsy ${ }^{24}$ and stroke, food and fluid restriction in macaque behavioral neuroscience ${ }^{25}$, and toxicology studies (for example, microsampling, social housing during cardiovascular recordings ${ }^{26}$ and body weight loss limits for acute studies ${ }^{27}$ ).

The expert working group on mammalian models of epilepsy, convened to review current practice and identify opportunities to improve animal welfare, was instigated at the request of academics from the epilepsy research community (http://www.nc3rs.org. uk/epilepsy). The strategy of the working group was multifaceted, incorporating a review of the scientific literature, a survey of the community, interviews with laboratory animal veterinarians and animal care staff, and the practical experience of the working group members. The group's report provides background information, practical guidance and recommendations for refinement, modelspecific advice and priorities for further research to facilitate best practice $^{24}$. The recommendations span the lifetime experience of animals used in epilepsy research, including seizure induction, maintenance and monitoring. Translation of research from animal models of epilepsy and seizures into the clinic has been highlighted as a major challenge ${ }^{28}$. Improving animal welfare and the quality of data from animal models maximizes their use and improves the translation of research to the clinic.

\section{Events and networks}

The NC3Rs raises awareness of opportunities for refinement by organizing regular scientific symposia and workshops, such as our annual meetings for users of non-human primates (Primate Welfare Meeting) and for animal technologists (Animal Technicians' Symposium) (http://www.nc3rs.org.uk/events); through supportive networks, such as our group for Named Animal Care and Welfare Officers (NACWOs) who work with non-human primates, and our wiki for neuroscientists using chronic implants (http:// www.ciwiki.net); and via our social media channels. We also have the funding capacity to sponsor events; for example, in July 2016, we sponsored a workshop on measuring animal emotions organized by grant holders Mike Mendl and Liz Paul. The workshop brought together experts in a range of animal emotion measures to discuss their utility, validity, strengths and weaknesses. In 2013, we funded an in international workshop on the welfare implications of different euthanasia methods for mice, rats and zebrafish. The report of the workshop summarizes the current understanding in the field and gives recommendations to help inform good practice for humane killing 29 .

We also have an annual 3Rs Prize (sponsored by GSK), which is awarded to recognize and highlight original scientific and technological advances in the 3 Rs published in the previous 3 years that have an effect on the use of animals in the life sciences. Previous winners for welfare-focused research with implications for scientific outcomes have been Laura Scullion Hall and collaborators, University of Stirling, for developing a framework for assessing the welfare of the laboratory beagle and validating refinements to the oral gavage dosing technique ${ }^{11}$; Brianna Gaskill and Joseph Garner, Purdue and Stanford Universities, for their work investigating the importance of nesting material and ambient temperature for thermoregulation in mice ${ }^{30}$; and Daniel Adams, University of California San Francisco, for taking inspiration from human orthopedics to develop a biocompatible, titanium head implant to reduce infection risk and improve welfare in monkeys undergoing brain recordings ${ }^{31}$.

\section{Online and other resources}

An extensive library of online resources is available on the NC3Rs website to provide researchers and technical staff with the practical tools needed to put refinement into practice (http://www.nc3rs. org.uk/3Rs-resources). These resources are used around the world and include information-rich websites with supporting references (for example, on the use and care of macaques and marmosets), high-definition video tutorials on the practical skills required to conduct common procedures humanely (for example, blood sampling, administration of substances, aseptic technique for rodent surgery) and e-learning resources that can be integrated into staff training in-house. The e-learning resources have been developed by Paul Flecknell and his team at Newcastle University with NC3Rs funding. They take a scenario-based learning approach to delivering the learning objectives of the training framework established under Directive $2010 / 63 / \mathrm{EU}^{32}$, with interactive problem solving in realistic contexts. The first three modules concern euthanasia of laboratory animals, anesthesia for minor procedures, and recognition and prevention of pain and distress.

There are ethical and legal obligations on researchers to avoid and alleviate pain in research animals ${ }^{33}$. However, some researchers argue that use of analgesic drugs will interfere with the variables they wish to study, such as cardiovascular function or nerve transmission. What is often not appreciated is that the animal's pain, and its reaction to that pain, may also confound the outcome of the experiment. Thus, good experimental design to eliminate unnecessary pain, through the use of anesthesia, analgesic drugs or nonpharmacological techniques, is scientifically important.

Effective alleviation of pain depends on the ability to recognize and assess its severity. Traditional methods of pain assessment, such as monitoring behavior and clinical signs, are time consuming and may not be specific to pain. Research by Jeffrey Mogil and his team at McGill University has demonstrated that changes in facial expression provide a reliable and rapid means of assessing pain in mice and rats ${ }^{34,35}$. 'Grimace scales' have been developed based on changes in a number of 'facial action units', such as narrowing of the eyes (orbital tightening) or changes in the position and shape 
of the whiskers. With funding from the NC3Rs, Matt Leach, also at Newcastle University, has validated the use of grimace scales for pain assessment in mice, rats and rabbits following surgical or other painful procedures ${ }^{36-38}$. To raise awareness about grimace scales and their potential to be used as part of clinical assessments ${ }^{39}$, the NC3Rs office has produced A3-sized posters of the mouse, rat and rabbit grimace scales for display in laboratory animal facility rooms. Over 16,000 posters in English, French and Chinese have been sent free of charge to laboratories in 51 countries.

\section{Conclusions}

At the NC3Rs, we recognize the importance of the link between good animal welfare and good quality science, as do many in the scientific community, but we are also involved in funding research that provides an evidence base for this relationship and in promoting this evidence base. Implementation of the opportunities that we have identified for refining research practices will improve not only animal welfare, but also scientific outcomes.

\section{COMPETING FINANCIAL INTERESTS}

The authors declare no competing financial interests.

\section{Received 10 October 2016; accepted 15 December 2016}

Published online at http://www.nature.com/laban

1. Clemence, M. \& Leaman, J. Public Attitudes to Animal Research in 2016. Ipsos MORI Social Research Institute/Department for Business, Energy and Industrial Strategy. http://www.ipsos-mori.com/Assets/Docs/ Publications/sri-public-attitudes-to-animal-research-2016.pdf (2016).

2. Home Office. Guidance on the Operation of the Animals (Scientific Procedures) Act 1986. HMSO. http://www.gov.uk/government/publications/ operation-of-aspa (2014).

3. NC3Rs, AMRC, BBSRC, Defra, EPSRC, MRC, NERC \& Wellcome Trust. Responsibility in the Use of Animals in Bioscience Research: Expectations of the Major Research Councils and Charitable Funding Bodies (NC3Rs, 2015).

4. Fleetwood, G. et al. Making progress and gaining momentum in global 3Rs efforts: how the European pharmaceutical industry is contributing. J. Am. Assoc. Lab. Anim. Sci. 54, 192-197 (2015).

5. Russel, W.M.S. \& Burch, R.L. The Principles of Humane Experimental Technique (Methuen, 1959).

6. Buchanan-Smith, H.M. et al. Harmonizing the definition of refinement. Anim. Welf. 14, 379-384 (2005).

7. Lloyd, M.H. et al. Refinement: promoting the three Rs in practice. Lab. Anim. 42, 284-293 (2008).

8. Poole, T. Happy animals make good science. Lab. Anim. 31, 116-124 (1997).

9. Mendl, M. \& Paul, E.S. Consciousness, emotion and animal welfare: insights from cognitive science. Anim. Welf. 13, S17-S25 (2004).

10. Mendl, M. et al. Cognitive bias as an indicator of animal emotion and welfare: emerging evidence and underlying mechanism. Appl. Anim. Behav. Sci. 118, 161-181 (2009).

11. Hall, L.E. et al. Refining dosing by oral gavage in the dog: a protocol to harmonise welfare. J. Pharmacol. Toxicol. Methods 72, 35-46 (2015).

12. Bethell, E. et al. Emotion evaluation and response slowing in a nonhuman primate: new directions for cognitive bias measures of animal emotion? Behav. Sci. 6, 2 (2016).

13. Ash, H. \& Buchanan-Smith, H.M. The long-term impact of infant rearing background on the affective state of adult common marmosets (Callithrix jacchus). Appl. Anim. Behav. Sci. 174, 128-136 (2016).

14. Gray, H. et al. Physiological, behavioral, and scientific impact of different fluid control protocols in the rhesus macaque (Macaca mulatta). eNeuro 3 (4), 1-15 (2016).
15. Scullion Hall, L.E.M. et al. The influence of facility and home pen design on the welfare of the laboratory-housed dog. J. Pharmacol. Toxicol. Methods 83, 21-29 (2017).

16. Holmes, A.M. et al. Impact of tank background on the welfare of the African clawed frog, Xenopus laevis (Daudin). Appl. Anim. Behav. Sci. 185, 131-136 (2016).

17. Hurst, J.L. \& West, R.S. Taming anxiety in laboratory mice. Nat. Methods 7, 825-826 (2010)

18. Gouveia, K. \& Hurst, J.L. Reducing mouse anxiety during handling: effect of experience with handling tunnels. PLOS ONE 8, e66401 (2013).

19. Tasker, L. Linking welfare and quality of scientific output in cynomolgus macaques (Macaca fasicularis) used for regulatory toxicology. PhD thesis, University of Stirling. http://dspace.stir.ac.uk/bitstream/1893/9801/1/ LouTaskerThesis2012.pdf (2012).

20. Tymvios, C. et al. Real-time measurement of non-lethal platelet thromboembolic responses in the anaesthetized mouse. Thromb. Haemost. 99, 435-440 (2008).

21. Moore, C. \& Emerson, M. Assessment of platelet aggregation responses in vivo in the mouse. Methods Mol. Biol. 788, 21-28 (2012).

22. Slater, H. et al. Individually customizable non-invasive head immobilization system for non-human primates with an option for voluntary engagement. J. Neurosci. Methods 269, 46-60 (2016).

23. Bains, R.S. et al. Analysis of individual mouse activity in group housed animals of different inbred strains using a novel automated home cage analysis system. Front. Behav. Neurosci. 10, 106 (2016).

24. Lidster, K. et al. Opportunities for improving animal welfare in rodent models of epilepsy and seizures. J. Neurosci. Methods 260, 2-25 (2016).

25. Prescott, M.J. et al. Refinement of the use of food and fluid control as motivational tools for macaques used in behavioral neuroscience research: Report of a working group of the NC3Rs. J. Neurosci. Methods 193, 167-188 (2010).

26. Prior, H. et al. Social housing of non-rodents during cardiovascular recordings in safety pharmacology and toxicology studies. J. Pharmacol. Toxicol. Methods 81, 75-87 (2016).

27. Chapman, K. et al. A global pharmaceutical company initiative: an evidence-based approach to define the upper limit of body weight loss in short term toxicity studies. Regul. Toxicol. Pharmacol. 67, 27-38 (2013).

28. Simonato, M. et al. The challenge and promise of anti-epileptic therapy development in animal models. Lancet Neurol. 13, 949-960 (2014).

29. Hawkins, P. et al. A good death? Report of the second Newcastle meeting on laboratory animal euthansia. Animals 6 (9), 50 (2016).

30. Gaskill, B.N. et al. Heat or insulation: behavioral titration of mouse preference for warmth or access to a nest. PLOS ONE 7, e32799 (2012).

31. Adams, D.L. et al. A watertight acrylic-free titanium recording chamber for electrophysiology in behaving monkeys. J. Neurophysiol. 106, 1581-1590 (2011).

32. National Competent Authorities for the implementation of Directive 2010/63/EU on the Protection of Animals Used For Scientific Purposes. A working document on the development of a common education and training framework to fulfil the requirements under the Directive: replacing consensus document of 18-19 September 2013, http://ec.europa.eu/ environment/chemicals/lab_animals/pdf/Endorsed_E-T.pdf (2014).

33. Carbone, L. Pain in laboratory animals: the ethical and regulatory imperatives. PLoS ONE 6, e21578 (2011).

34. Langford, D.J. et al. Coding of facial expressions of pain in the laboratory mouse. Nat. Methods 7, 447-449 (2010).

35. Sotocinal, S.G. et al. The Rat Grimace Scale: a partially automated method for quantifying pain in the laboratory rat via facial expressions. Mol. Pain 7, 55. 10.1186/1744-8069-7-55 (2011).

36. Leach, M.C. et al. The assessment of post-vasectomy pain in mice using behavior and the Mouse Grimace Scale. PLOS ONE 7, e35656 (2012).

37. Keating, S.C.J. et al. Evaluation of EMLA cream for preventing pain during tattooing of rabbits: changes in physiological, behavioral and facial expression responses. PLOS ONE 7, e44437 (2012).

38. Miller, A.L. et al. The influence of isoflurane anaesthesia on the Rat Grimace Scale. PLOS ONE 11, e0166652 (2016).

39. Leung, V. et al. Real-time application of the Rat Grimace Scale as a welfare refinement in laboratory rats. Sci. Rep. 6, 31667 (2016). 